\title{
Reconstruction of Severely Degraded Image Sequences ${ }^{\star}$
}

\author{
ANIL C. KoKARAM \\ Signal Processing and Communications Group, \\ Engineering Dept., University of Cambridge, \\ Trumpington St., Cambridge CB2 1PZ \\ England. \\ ack@eng . cam. ac . uk \\ Tel : +44 1223332767, Fax : +44 1223332662
}

\begin{abstract}
The AURORA project (AUtomated Restoration of ORiginal Film Archives) is an E.U. funded ACTS project which began in September of 1996. The partners include three broadcasters and holders of archives, The Insitut National L'Audiovisuel (INA), The British Broadcasting Corporation, Radiotelevisao Portuguesa, two industrial companies, Snell and Wilcox, Societe Generale de Teleinformatic, and the signal processing groups of three academic institutions the Digital Media Institute, (Tampere, Finland) Delft University (The Netherlands) and Cambridge University Engineering Dept. (U.K). The project, coordinated by INA, has the sole purpose of designing new tools for video restoration/enhancement. This goal is motivated by the lack of a complete set of advanced manipulation tools which would otherwise allow the more complete exploitation of the archive holdings of many of the larger broadcasters. Furthermore, with the oncoming rise in Digital Video broadcasting a higher demand on quality and quantity of archive material is perceived; hence the requirement for real time restoration devices is set to become more exacting. The project therefore considers the usual cornerstones of video restoration : noise reduction, missing data detection and reconstruction, reduction of image unsteadiness; as well as the associated software and hardware implementation issues. This paper concentrates on new developments at Cambridge University with respect to missing data reconstruction using probabilistic formulations.
\end{abstract}

Missing data is a common impulsive degradation in archived film. The film material is abraded or occluded by foreign material caught in the projection mechanism thus yielding bright and dark flashes of light : blotches. The treatment of missing data in image sequences has traditionally been achieved by the use of spatio-temporal median filters. $[1,2]$ presented a series of alternatives which illustrate the need for a detector to control the operation of an interpolator which may take the form of a rank-order operation or employ a model based constraint on the spatio-temporal evolution of the image sequence. Within an interpolation framework, the spatio-temporal AR model was shown to be extremely accurate at reconstructing texture in missing regions as large as $20 \times 20$

* Work funded under E.U. contract AC072, AURORA. 
pixels. However, in order to extend the technique to deal with larger missing regions (up to the entire frame : see fig. 2) it is necessary to design a non-stationary interpolation scheme which enables the AR model to adapt to the underlying non-stationary nature of typical scenes. While it is possible to develop such algorithms, it becomes more difficult to control the performance of the interpolation mechanism. Recent work has concentrated instead on stochastic techniques for reconstructing the motion and occlusion field in the missing region. Using this information, relatively simple interpolation schemes may then be employed for reconstructing the missing regions. The following sections highlight the key points in the presentation of new priors for occlusion and the novel use of cooperative deterministic and stochastic schemes to solve the huge multivariable problem which is proposed.

\section{A Bayesian Framework for Motion and Image Field Reconstruction}

Given a region denoted as missing it is required to estimate both the motion field and discontinuity information associated with that missing patch. Figure 2 shows the kind of large missing area which is concerned. In order to proceed, an occlusion parameter $o_{k, k-1}(\mathbf{x})$ is defined which is set to $1(\mathrm{ON})$ if the data in frame $k$ at position $\mathbf{x}$ is obscured in frame $k-1 ; o_{k, k+1}(\mathbf{x})$ is 1 if the data in frame $k$ is obscured in $k+1$; and 0 otherwise. It is further assumed that the image model is translational such that $I_{n}(\mathbf{x})=I_{n-1}\left(\mathbf{x}+\mathbf{d}_{n, n-1}(\mathbf{x})\right)+v_{n}(\mathbf{x})$, where $v_{n}(\mathbf{x}) \sim N\left(0, \sigma_{v v}^{2}\right)$, and $\mathbf{d}_{n, n-1}$ is the motion vector mapping frame $n$ into $n-1$. The known location of a missing pixel is denoted as $\mathbf{x}_{u}$, and the unknown intensity at that location in frame $\mathrm{k}$ as $I_{k}\left(\mathbf{x}_{u}\right)$.

Ultimately, the problem is to estimate a value for $I_{k}\left(\mathbf{x}_{u}\right)$. This must implicitly involve solving for motion information from several frames, since the underlying image model is primarily temporal. As quantification of temporal discontinuities are an important part of rendering sharp interpolants, these parameters must also be estimated. An estimate for the parameters at each pixel site would be generated by maximizing

$$
p\left(I_{n}\left(\mathbf{x}_{u}\right), \mathbf{d}_{n, n-1}\left(\mathbf{x}_{u}\right), \mathbf{d}_{n, n+1}\left(\mathbf{x}_{u}\right), o_{n, n-1}\left(\mathbf{x}_{u}\right), o_{n, n+1}\left(\mathbf{x}_{u}\right) \mid D, O, I_{n-1}, I_{n+1}\right)
$$

with respect to the two sets of motion and occlusion parameters as well as the unknown intensity. Here $D, O$ denotes the local neighbourhoods of motion and occlusion parameters. Optimal solution for the parameters over the entire missing region would necessarily involve the implementation of the Gibbs sampler $[3,4]$ in the usual way, relaxing the entire system until convergence.

Employing Bayes' theorem allows an expression for the joint posterior to be written as

$$
\begin{aligned}
p\left(I_{n}\left(\mathbf{x}_{u}\right), \mathbf{d}_{n, n-1}\left(\mathbf{x}_{u}\right), o_{n, n-1}\left(\mathbf{x}_{u}\right) \mid D, O, I_{n-1}, I_{n+1}\right) \propto \\
p\left(I_{n-1} \mid I_{n}\left(\mathbf{x}_{u}\right), \mathbf{d}_{n, n-1}\left(\mathbf{x}_{u}\right), o_{n, n-1}\left(\mathbf{x}_{u}\right), I_{n+1}, D, O\right)
\end{aligned}
$$




$$
\begin{aligned}
& \times p\left(\mathbf{d}_{n, n-1}\left(\mathbf{x}_{u}\right) \mid o_{n, n-1}\left(\mathbf{x}_{u}\right), I_{n+1}, D, O\right) \\
& \times p\left(o_{n, n-1}\left(\mathbf{x}_{u}\right) \mid I_{n+1}, D, O\right)
\end{aligned}
$$

The expression has been simplified to solve for three of the parameters at a time instead of all five. The result for the remaining variable pair

$\mathbf{d}_{n, n+1}\left(\mathbf{x}_{u}\right), o_{n, n+1}\left(\mathbf{x}_{u}\right)$ can be derived similarly, with $D, O$ being augmented by the relevant spatial/temporal neighborhood. The joint posterior consists of a likelihood term and two priors on motion and occlusion smoothness. Having derived reasonable functional forms for the various distributions, the Gibbs sampler may be used to locate the MAP estimate. This is obviously a huge solution space and convergence is unlikely to be achieved in a practical time. However, it is possible to manipulate the posterior to sample for several unknowns jointly. Furthermore it is possible to draw upon the near-success of deterministic schemes to effectively kick start the Gibbs sampler and achieve a practical process. These ideas are introduced later on in this paper. The sections immediately following describe the functional forms of the various probability distributions required and introduce a new prior for occlusion estimation.

THE LIKELIHOOD : It is typical to associate the presence of an ON occlusion site with the violation of temporal image smoothness $[5,6]$, implicit in the image model proposed above. This would therefore involve the inclusion of an occlusion variable in the expression for the data likelihood. However, closer investigation of the behaviour of motion fields with respect to a moving object (see figure 1) indicates that occlusion can also be flagged due to a violation of a mirror constraint with respect to motion vectors. This constraint is more powerful than the intensity constraint, for the estimation of the occlusion fields. Therefore, the data likelihood is derived directly from the image model as

$$
\begin{aligned}
p\left(I_{n-1} \mid I_{n}\left(\mathbf{x}_{u}\right), \mathbf{d}_{n, n-1}\left(\mathbf{x}_{u}\right), o_{n, n-1}\left(\mathbf{x}_{u}\right), I_{n+1}, D, O\right) \propto \\
\exp \left(-\frac{1}{2 \sigma_{v v^{2}}}\left(I_{n}\left(\mathbf{x}_{u}\right)-I_{n-1}\left(\mathbf{x}+\mathbf{d}_{n, n-1}(\mathbf{x})\right)^{2}\right)\right.
\end{aligned}
$$

THE MOTION PRIOR : It is clear that spatially, the motion field should be smooth. In this paper, spatial discontinuities are omitted for clarity, but they can be included into the final framework. The spatial component of the motion field prior is kept simple, and the usual Gibbs energy distribution is employed. It is of course independent of $I, O$.

$$
\begin{aligned}
& p\left(\mathbf{d}_{n, n-1}(\mathbf{x}) \mid D\right) \propto p\left(\mathbf{d}_{n, n-1}(\mathbf{x}) \mid \mathbf{d}_{n-1, n}, o_{n}(\mathbf{x})\right) \\
& \quad \times \exp -\left(\sum_{\mathbf{s} \in L} \lambda_{\mathbf{s}}\left|\mathbf{d}_{n, n-1}(\mathbf{x})-\mathbf{d}_{n, n-1}(\mathbf{x}+\mathbf{s})\right|\right)
\end{aligned}
$$

Here $L$ denotes the support region of the vector neighborhood (the $3 \times 3$ local neighborhood is used), and $\mathbf{s}$ indexes each of the positions within this region as usual. The first term represents temporal smoothness. 
It is through temporal smoothness that a new motion prior may be introduced.

$$
\begin{aligned}
& p\left(\mathbf{d}_{n, n-1}(\mathbf{x}) \mid d_{n-1, n}, o_{n}(\mathbf{x})\right) \propto \\
& \quad \exp -\left(\frac{1}{2 \sigma_{d d^{2}}}\left|\mathbf{d}_{n, n-1}(\mathbf{x})-\mathbf{d}_{n-1, n}\left(\mathbf{x}+\mathbf{d}_{n, n-1}(\mathbf{x})\right)\right|^{2}\left(1-o_{n}(\mathbf{x})\right)\right)
\end{aligned}
$$

This is a statement of the well known phenomenon illustrated in figure 1. Setting the occlusion $\mathrm{ON}$ in equation 4 turns off the contribution of this term. The occlusion prior must therefore be designed to penalize the presence of ON occlusion states.

THE OCCLUSION PRIOR : This term also consists of two parts. The spatial part is identical to the form used for spatial motion smoothness and is not detailed here. The second part is a penalty term which can be related to the expected smoothness of the motion field. Thus $p\left(o_{n, n-1}(\mathbf{x})\right) \propto \exp -\left(\alpha o_{n, n-1}(\mathbf{x})\right)$. The penalty $\alpha$ is therefore introduced whenever occlusion is ON. This penalty must balance the degree of violation of the mirror constraint and acts as a switch which employs occlusion when this violation becomes intolerable. Since the temporal motion smoothness is assumed to be governed by $N\left(0, \sigma_{d d}^{2}\right)$, it is simple to calculate normalized confidence limits on $\left|\mathbf{d}_{n, n-1}(\mathbf{x})-\mathbf{d}_{n-1, n}\left(\mathbf{x}+\mathbf{d}_{n, n-1}(\mathbf{x})\right)\right|^{2}$. This then gives immediately a reasonable value for $\alpha$. For instance to be $99 \%$ confident that a motion vector has violated the normal assumption as far as temporal smoothness is concerned, $\alpha=2.576^{2} / 2$.

\section{Stochastic Optimization}

Having assembled the various terms together it is now necessary to solve the system for all the unknown parameters. The hyperparameters $\sigma_{v v}, \sigma_{d d}, \alpha$ could be estimated together with the unknowns in a Gibbs sampler. However, this will obviously degrade the convergence of the final algorithm, therefore reasonable estimates are made. The previous section introduced a simple mechanism for choosing $\alpha$. In addition, $\sigma_{v v}$ could be measured from the image data in stationary regions. $\sigma_{d d}$ cannot be simply measured and its choice must therefore be based on experience of the expectation of motion smoothness.

Denoting the vector of all the parameters excepting one by e.g $\mathbf{P}_{-I}$ which excepts the image data, the direct use of the Gibbs sampler involves the derivation of conditional likelihoods of the form $p\left(I_{n}\left(\mathbf{x}_{u}\right) \mid \mathbf{P}-I\right), p\left(\mathbf{d}_{n, n-1} \mid \mathbf{P}_{-d}\right)$ etc. These may be derived from the joint posterior shown in equation 2 . The sampler then operates iteratively, given some starting guess for the unknowns, by drawing random samples from the conditional posterior distribution for each unknown in turn :

$$
\begin{aligned}
I_{n}\left(\mathbf{x}_{u}\right) & \sim p\left(I_{n}\left(\mathbf{x}_{u}\right) \mid d_{n, n-1}\left(\mathbf{x}_{u}\right), o_{n, n-1}\left(\mathbf{x}_{u}\right), D, O\right) \\
\mathbf{d}_{n, n-1}\left(\mathbf{x}_{u}\right) & \sim p\left(\mathbf{d}_{n, n-1}\left(\mathbf{x}_{u}\right) \mid I_{n}\left(\mathbf{x}_{u}\right), o_{n, n-1}\left(\mathbf{x}_{u}\right), D, O\right) \\
o_{n, n-1}\left(\mathbf{x}_{u}\right) & \sim p\left(o_{n, n-1}\left(\mathbf{x}_{u}\right) \mid I_{n}\left(\mathbf{x}_{u}\right), \mathbf{d}_{n, n-1}\left(\mathbf{x}_{u}\right), D, O\right)
\end{aligned}
$$


For a typical video sequence, the motion can be very large. This implies that a scheme which relies on the convergence of a Gibbs Sampler starting from a zero estimate of the parameters will be extremely impractical. It is advantageous to consider the use of a deterministic multiresolution motion estimator to provide initial estimates for the sampler. Unfortunately, this may not be enough. The missing region may be so large as to make the output of any deterministic motion estimator useless. This is the situation in figure 2. However, some interesting approximations help to dispel this apparent hopelessness.

First of all, if it is assumed that frames $n-1, n+1$ are relatively uncorrupted in the regions which are corrupted in frame $n$, then it is reasonable to assume that the vectors e.g. $\mathbf{d}_{n-1, n+1}, \mathbf{d}_{n+1, n-1}$ can be correctly estimated with a deterministic motion estimation stage. Assuming low acceleration implies that $\mathbf{d}_{n, n-1} \approx \mathbf{d}_{n+1, n-1} / 2$, suitably compensated for motion. Thus at any site $\mathbf{x}_{u}$ it is possible to propose a set of temporal motion vectors, derived from the outer two frames, which may be used to start the Gibbs sampler, see figure 1. This set may be augmented by spatial candidates from the already configured motion estimates in frame $n$ as the algorithm proceeds. Furthermore, it is possible to split the process into a motion/occlusion configuration stage and an image interpolation stage. This can be done by employing the image model to approximate $I_{n}\left(\mathbf{x}_{u}\right)-I_{n-1}\left(\mathbf{x}_{u}+\mathbf{d}_{n, n-1}\left(\mathbf{x}_{u}\right)\right)$ by $I_{n-1}\left(\mathbf{x}_{u}+\mathbf{d}_{n, n-1}\left(\mathbf{x}_{u}\right)-I_{n+1}\left(\mathbf{x}_{u}+\mathbf{d}_{n, n+1}\left(\mathbf{x}_{u}\right)\right)\right.$.

Assuming further that the optimal interpolated motion vector is one of the candidates selected from the surrounding frames as discussed previously, then it is possible to numerically sample from this vector subset rather than resorting to Metropolis techniques. The sampling is done through the direct numerical evaluation of the probabilities involved. Towards increased utility, it is possible to employ the method of composition [4] to sample jointly for the motion and occlusion parameters at each pixel site visited. A random draw from the density $p(\mathbf{d}, \mathbf{o} \mid \theta)$, for instance, is made possible by the decomposition $p(\mathbf{d}, \mathbf{o} \mid \theta)=p(\mathbf{o} \mid \mathbf{d}, \theta) p(\mathbf{d} \mid \theta)$, where $p(\mathbf{d} \mid \theta)$ is derived by integrating out $\mathbf{o}$ from the joint posterior. In this case the decomposition is trivial since the occlusion is a binary variable. To draw a joint sample therefore, $\mathbf{d}_{n, n-1}$ is drawn from $p\left(\mathbf{d}_{n, n-1} \mid O, D, I\right)$ then this sample is employed in the draw $o_{n, n-1} \sim p\left(o_{n, n-1} \mid \mathbf{d}_{n, n-1}, O, D, I\right)$.

\section{Results and Final Comments}

Figure 2 shows three frames from a severely degraded sequence. The central frame has been subject to digital dropout, and a major portion has been corrupted. The sequence shows large motion in the arm of the man and a simultaneous pan and zoom. Figure 3 shows the restoration achieved ${ }^{2}$ after 10 iterations of the sampler using a kick start from the motion estimation scheme described in [7], $\sigma_{v v}^{2}=20.0, \sigma_{d d}=4.0$, and $\alpha=2.576^{2} / 2$. Joint sampling for $\mathbf{d}, O$ was employed, but no edge field prior was used.

The pictures illustrate the power of a scheme which employs both deterministic and stochastic mechanisms for solving an ill-posed problem. The hybrid process relies on the ability of standard motion estimation schemes to achieve

${ }^{2}$ The results of the colour version of this algorithm can be seen at http://ww-sigproc.eng.cam.ac.uk/ ack/. 
"almost accurate" estimates. The subsequent stage is then allowed the freedom to employ a more complete model of the problem while still being able to achieve a practical convergence rate. The interpolated result shows a dramatic improvement in picture quality.

\section{References}

1. A. Kokaram, R. Morris, W. Fitzgerald, and P. Rayner. Detection of missing data in image sequences.. IEEE Image Processing, pages 1496-1508, Nov. 1995.

2. A. Kokaram, R. Morris, W. Fitzgerald, and P. Rayner. Interpolation of missing data in image sequences.. IEEE Image Processing, pages 1509-1519, Nov. 1995.

3. S. Geman and D. Geman. Stochastic relaxation, gibbs distributions and the bayesian restoration of images. IEEE PAMI, 6:721-741, 1984.

4. J.J. O Ruanaidh and W. J. Fitzgerald. Numerical Bayesian Methods Applied to Signal Processing. Springer Verlag, Springer Series in Statistics and Computing, 1996.

5. S. Z. Li. Markov Random Field Modelling in Computer Vision. Springer Verlag, 1995.

6. R. D. Morris. Image Sequence Restoration using Gibbs Distributions. PhD thesis, Cambridge University, England, 1995.

7. A. Kokaram and S. Godsill. A system for reconstruction of missing data in image sequences using sampled 3D AR models and MRF motion priors. In European Conference on Computer Vision 1996, pages 613-624. Springer Verlag, April 1996.
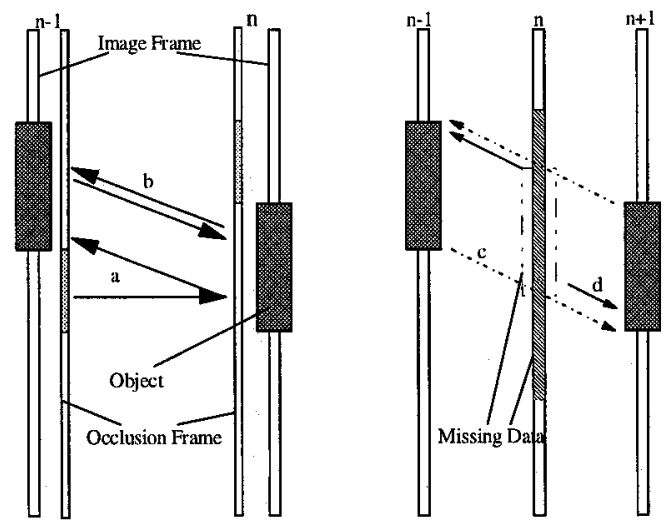

Fig. 1. Left : Violation of mirror constraint illustrated in region $a$. Mirror pair indicating no occlusion shown in region $b$. The occlusion frame is shaded where it is ideally set to 1 . Right : Use of deterministic motion estimates $(c)$ across the missing frame (dashed arrows), to generate start candidates $(d)$ for the sampler. 


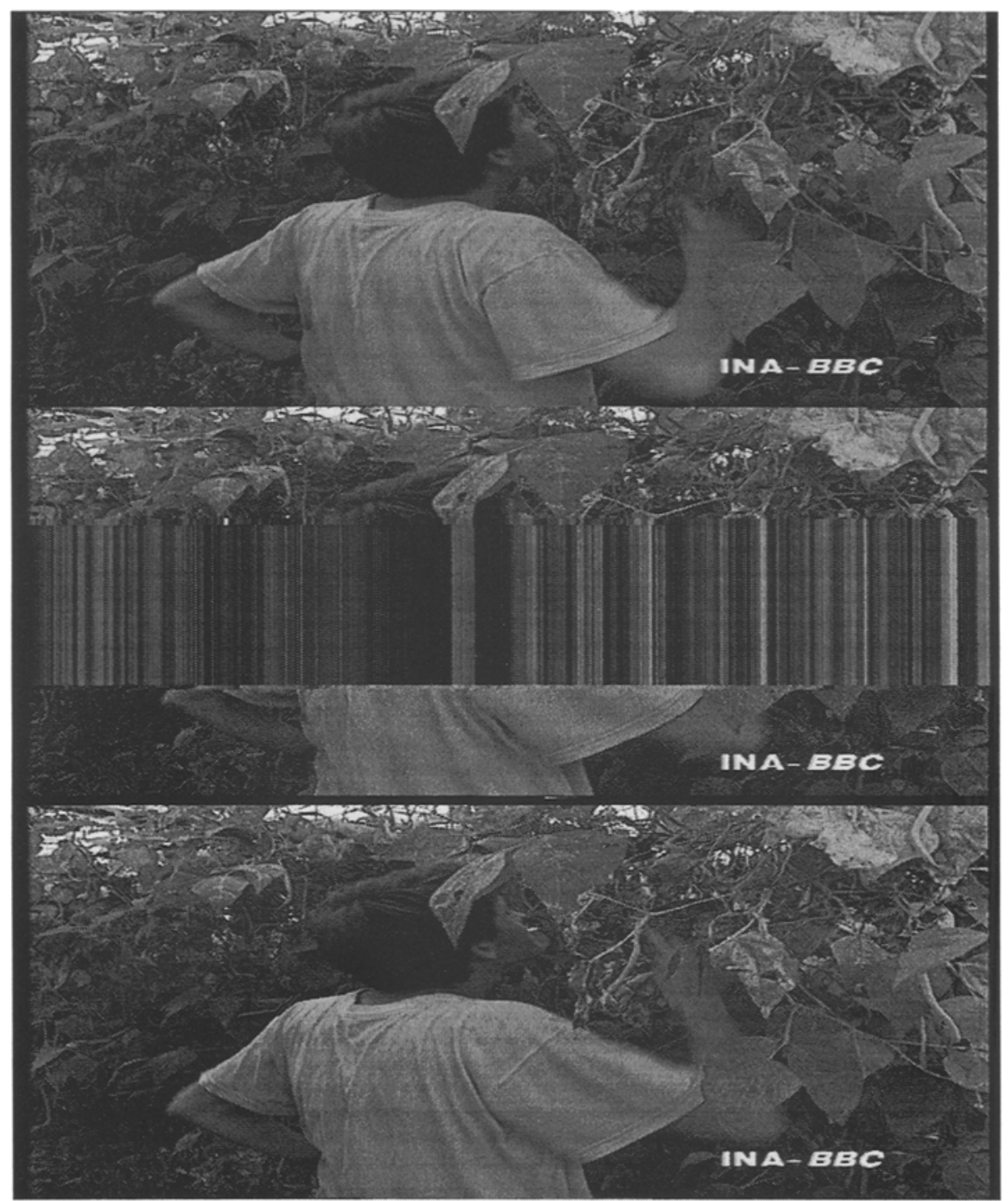

Fig. 2. Top-bottom : Frames 1-3 of a badly degraded sequence. Centre frame shows huge errors. 


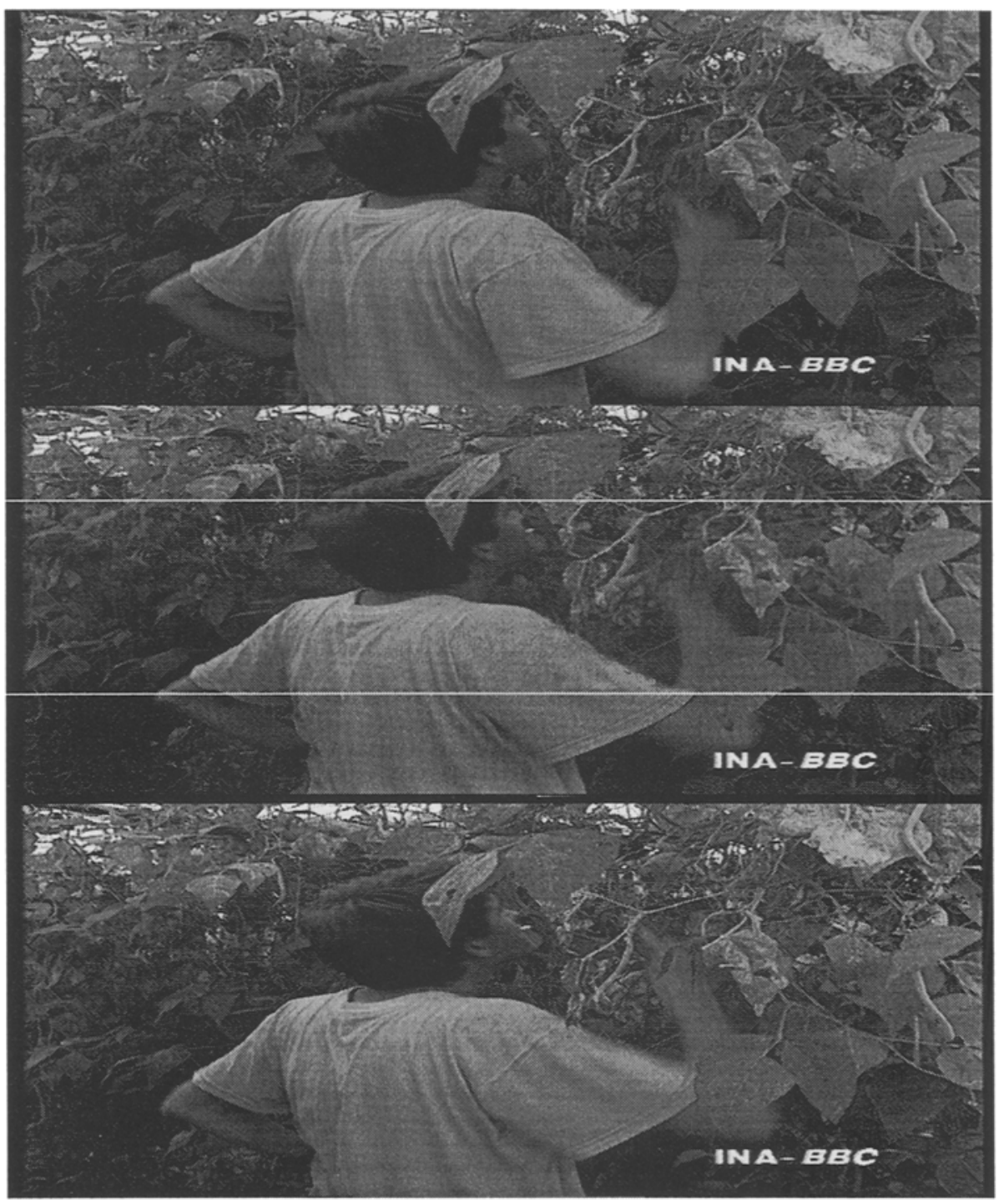

Fig. 3. Top-bottom : Restored central frame inserted in the degraded sequence. The interpolated area is boxed. The discontinuity at the top and bottom edge of the interpolated area is due to picture shake caused by the degradation process in the central frame. Compensation for this is not part of the interpolation process. 